\title{
Cálculo de eficiência na alocação de mão de obra em estações ferroviárias da malha do Rio de Janeiro por meio da análise envoltória de dados (DEA)
}

\author{
Luíza Serra Moreira' ${ }^{1}$, Wagner Luis Ferreira², João Carlos Correia Baptista Soares de Mello ${ }^{3}$ \\ 1Universidade Federal Fluminense, UFF, luizaserramoreira@id.uff.br \\ 2Universidade Federal Fluminense, UFF, w.ferreira@globo.com \\ 3Universidade Federal Fluminense, UFF, jcsmello@producao.uff.br
}

\section{Recebido:}

28 de março de 2018

Aceito para publicação:

9 de dezembro de 2018

Publicado:

31 de dezembro de 2019

Editor de área:

Renato Lima

\section{Palavras-chaves:}

Eficiência,

Ferrovia,

Análise envoltória de dados.

Keywords:

Efficiency,

Railway,

Data envelopment analysis.

DOI:10.14295/transportes.v27i4.1644

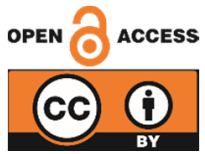

\begin{abstract}
RESUMO
O presente objeto de estudo analisa a eficiência em relação a utilização da mão de obra e passageiros atendidos nas estações ferroviárias do principal ramal ferroviário da cidade do Rio de Janeiro. $\mathrm{O}$ objetivo é de viabilizar uma análise de melhores práticas nas estações, possibilitando uma melhor alocação de mão de obra. Para tanto, foi utilizado o DEA como ferramenta de elaboração dos cálculos, por meio do modelo CCR com orientação a inputs e análise da fronteira invertida. Os dados iniciais adotados correspondem ao total de funcionários (input) estimados por meio de observações, e as quantidades de passageiros por estação (output) que correspondem à média de dias úteis entre maio de 2016 até maio de 2017 multiplicados por um fator de correção. A seleção de outras variáveis, por vez, foi realizada utilizando o método de Multicritério para Seleção de Variáveis. Além disso, uma ampla revisão de literatura foi pesquisada como referências aos conceitos e premissas aqui adotados. $O$ resultado apresentou a estação de São Cristóvão como eficiente a ponto de ser considerada benchmark. Dessa forma, as práticas e critérios adotados na utilização de mão de obra na estação de São Cristóvão devem ser, quando operacionalmente possível, replicados e/ou adaptados às demais estações.
\end{abstract}

\section{ABSTRACT}

This case study analyzes the efficiency in relation to the utilization of labor and passengers served from the railway stations of the main railway branch of the Rio de Janeiro city. The objective is to make feasible an analysis of best practices in the stations, allowing a better allocation of labor. For this purpose, the DEA was used as a computation tool, using the CCR model with input orientation and inverted border analysis. The initial data used correspond to the total number of employees (input) estimated by observation, and the number of passengers per station corresponds to the average number of business days between May 2016 and May 2017 multiplied by a correction factor. The selection of other variables was performed using the Multicriteria Method for Selection of Variables. In addition, a large literary revision was made as references to the concepts and premises adopted here.

\section{INTRODUÇÃO}

As estações ferroviárias podem ser consideradas o principal meio de interface entre passageiros e a via férrea uma vez que, por meio delas, ocorrem a entrada de usuários no sistema ferroviário, embarque e desembarque nos vagões e mudanças de linha. De acordo com Sameni et al. (2016), estações usualmente são gargalos da rede ferroviária. Contudo, poucos trabalhos são dedicados a avaliar e ranquear suas perfomances. 
Por apresentar custos de operação e manutenção elevados, faz-se necessária a melhor utilização dos recursos disponíveis, tais como materiais, energia e mão de obra. Neste estudo serão analisadas as eficiências relativas à utilização de mão de obra nas estações ferroviárias do Ramal de Deodoro, no Rio de Janeiro, relacionadas à quantidade de passageiros e ao número de linhas atendidas utilizando a ferramenta de Análise Envoltória de Dados (DEA). A análise tem como objetivo apoiar possíveis tomadas de decisões futuras em relação a melhor utilização da mão de obra disponível no que se relaciona ao quadro de funcionários e à alocação de trabalhadores nas estações, apontando as unidades eficientes que possuem melhores práticas e podem se tornar benchmarks para as demais. Para atingir esse objetivo, além da presente introdução, este artigo adota a seguinte estrutura: Revisão de Literatura, Análise Envoltória de Dados (DEA), Método de Pesquisa, Objeto de Estudo e Conclusões.

\section{REVISÃO DE LITERATURA}

\subsection{Malha ferroviária do Rio de Janeiro}

O transporte público é um grande desafio para a locomoção de milhares de cidadãos nos grandes centros urbanos. Os números do sistema ferroviário do Rio de Janeiro demonstram sua dimensão e complexidade: extensão de 270 km, 102 estações, 5 ramais e 3 extensões, servindo 12 municípios da RMRJ e transportando 700 mil passageiros por dia útil, com 201 trens em operação.

\subsection{DEA aplicado em Ferrovias}

A fim de embasar o estudo, foram pesquisados diversos trabalhos que envolvem a aplicação de DEA em ferrovias. Entre eles estão os apresentados a seguir.

Oum e Yu (1994) realizam uma análise utilizando DEA a fim de identificar os efeitos das intervenções governamentais e subsídios na eficiência produtiva dos sistemas ferroviários que possuem grande parte de seus serviços voltados para passageiros em países Europeus e Asiáticos. Utiliza como inputs o número de funcionários, o consumo de energia, a estrutura, materiais, número de vagões de passageiros, número de vagões de carga e número de locomotivas. As variáveis utilizadas como outputs são número de passageiros por $\mathrm{Km}$, toneladas por $\mathrm{Km}$, trem de passageiros por Km e trem de carga por Km.

Lan e Lin (2003) utilizam DEA orientado a input para investigar a eficiência técnica e a efetividade do serviço de 76 ferrovias ao redor do mundo no período de 1999-2001. Utilizam como inputs a extensão da rede, a quantidade de locomotivas e vagões e o número de empregados. Como output utilizam apenas a variável trem por Km.

Yu (2008) utilizou os modelos DEA, TDEA (Traditional Data Development Analysis) e NDEA (Network Data Development Analysis) visando comparar a eficiência de 40 ferrovias. Os inputs utilizados foram comprimento da linha, número de funcionários, número de vagões de carga e número de vagões de passageiros, e como outputs foram utilizados tonelada por Km e passageiros por Km.

Himola (2010) apresenta em seu artigo o modelo DEA multiestágio para analisar a eficiência operacional e financeira do modo ferroviário de alguns países. Para eficiência operacional, os inputs utilizados foram a quilometragem da malha, o número de vagões, de locomotivas e o número de funcionários, e os outputs considerados foram a tonelada quilômetro útil e a tonelada útil tracionada. Por outro lado, o cálculo da eficiência financeira utilizou como inputs as saídas da modelagem da eficiência operacional, e como outputs a receita e o frete. 
Caldas et al. (2012) aplicam o modelo DEA CCR orientado a output para comparar a eficiência operacional das concessionárias brasileiras e americanas. Utilizam como entradas a extensão da linha em quilômetros e o número de funcionários trabalham no trecho. 0 output foi a quantidade de carga transportada no trecho (em toneladas).

Kutlar et al. (2013) mediram a eficiência de 31 ferrovias de diversos países, de 2000 a 2009. Foram considerados como inputs o número de funcionários, comprimento da linha, número de vagões de carga, número de vagões de passageiros, número de veículos de operação e custo da operação anual. 0 estudo considerou como outputs a tonelada por quilômetro, passageiros por quilômetro, tonelada transportada, número de passageiros transportados e receita anual.

Pereira e Soares de Mello (2014) estudam a eficiência das concessionárias brasileiras nos anos de 2009 a 2011 por meio do modelo DEA BCC orientado a output. Os inputs utilizados foram a meta de carga transportada e a extensão da malha concessionada, enquanto que os outputs foram a carga real transportada e a receita.

Pereira et al. (2015) analisam a eficiência das empresas concessionárias da malha ferroviária brasileira, no período de 2009 a 2013. Nesta análise são utilizados como inputs a área de concessão, número de empregados, gastos com pessoal, gastos com depreciação, outros gastos e total de gastos, bem como a receita líquida é utilizada como output.

Reis et al. (2017) estudaram a eficiência de ferrovias utilizadas para transporte de cargas utilizando métodos de seleção de variáveis e outputs indesejáveis. Utilizando o modelo BCC orientado a output, foram utilizados como inputs os investimentos e o número de acidentes e, como outputs, a carga transportada e o número de clientes.

Sameni et al. (2016) realizam análise de 96 estações ferroviárias da Grã Bretanha em relação a eficiência das mesmas e a efetividade do serviço. Para isso, desenvolveram o modelo de avaliação de eficiência técnica orientado a output utilizando o número de paradas que os trens realizam nas estações (informação retirada da grade horária) e como inputs o número de plataformas e porcentagem de linhas de passagem em cada estação (calculada divindindo o total de linhas de passagem sobre a soma do total de linhas de passagem e total de linhas terminais em cada estação). Já para a análise de efetividade do serviço foram realizadas análises orientadas tanto a output como a input. Dessa forma, as variáveis de entrada foram o número de paradas que os trens realizam nas estações, a área populacional captada e o número de oportunidades de trabalho na área captada. Já as variáveis de saída utilizadas foram o número de passageiros que entram e saem nas estações e o número de mudanças de linhas realizadas pelos usuários.

\section{ANÁLISE ENVOLTÓRIA DE DADOS (DEA)}

A Análise Envoltória de Dados (DEA - Data Envelopment Analysis), desenvolvida por Charnes et al. (1978), é uma ferramenta utilizada para análise de eficiência de unidades produtivas ou tomadoras de decisão (DMUs - Decision Making Units). Por meio de uma abordagem não-paramétrica baseada em modelos de programação matemática, as eficiências das DMUs são calculadas individualmente comparando as quantidades produzidas (outputs) em relação aos recursos utilizados (inputs) aos níveis de todas as outras unidades envolvidas no estudo.

0 método DEA otimiza cada observação individual objetivando calcular uma fronteira de eficiência, determinada pelas unidades que são Pareto Eficientes, ou seja, que não conseguem melhorar alguma de suas características sem deteriorar as demais. Como resultado, tem-se a construção de uma fronteira eficiente. As DMUs que se encontram na fronteira são consideradas 
eficientes, que tem sua pontuação atribuída a 100\%, enquanto as demais são consideradas ineficientes. Dessa forma, as DMUs na fronteira se tornam benchmarks para as demais, ou seja, se tornam referência em relação a níveis de inputs, outputs e práticas gerenciais.

Em geral, há duas formas básicas de DMUs não eficientes alcançarem a fronteira. A primeira é por meio da orientação a input, que parte do pressuposto da redução dos recursos utilizados mantendo os mesmos níveis de produção. Já a orientação a output visa o aumento das quantidades produzidas, mantendo constantes os recursos utilizados (inputs).

DEA apresenta dois modelos clássicos: CCR e BCC. 0 primeiro, proposto por Charnes et al. (1978), também é denominado por CRS (Constant Returns to Scale), uma vez que trabalha com retornos constantes de escala. Dessa forma, toda variação nas entradas implica em variação proporcional nas saídas, obedecendo, portanto, a propriedade do raio ilimitado (Cooper et al., 2007).

Esse modelo maximiza o quociente entre a combinação linear dos outputs e a combinação linear dos inputs, com a restrição de que para qualquer DMU esse quociente não pode ser maior que 1. O modelo CCR dos multiplicadores é apresentado pelas equações (1), (2) e (3) que formam o Problema de Programação Linear (PPL):

$$
\operatorname{Max} E f f_{o}=\sum_{\mathrm{j}=1}^{\mathrm{s}} \mathrm{u}_{\mathrm{j}} \mathrm{y}_{\mathrm{jo}}
$$

Sujeito a:

$$
\begin{gathered}
\sum_{i=1}^{r} v_{i} x_{i o}=1 \\
\sum_{j=1}^{s} u_{j} y_{j k}-\sum_{i=1}^{r} v_{i} x_{i k} \leq 0, k=1, \ldots, n \\
u_{j}, v_{i} \geq 0, i=1, \ldots, s, j=1, \ldots, r
\end{gathered}
$$

em que Effo: eficiência da DMU o em análise

vi e $u j$ : $\quad$ pesos de inputs $\mathrm{i}, \mathrm{i}=1, \ldots, \mathrm{r}$, e outputs $\mathrm{j}, \mathrm{j}=1, \ldots, \mathrm{s}$ respectivamente

xik e yjk: inputs i e outputs $\mathrm{j}$ da DMU $\mathrm{k}, \mathrm{k}=1, \ldots, \mathrm{n}$

xio e yjo: inputs i e outputs j da DMU o

0 modelo dos multiplicadores é primordialmente utilizado para cálculo de eficiências e dos pesos das variáveis. Para o cálculo dos benchmarks utiliza-se a modelagem dual do PPL conhecida como Modelo Envelope e exposto nas equações (4), (5) e (6):

Sujeito a:

$$
\begin{gathered}
\text { Min Effo }=h \\
h x_{i o}-\sum_{k=1}^{n} x_{i k} \lambda_{k} \geq 0, \forall i
\end{gathered}
$$

$$
-y_{j o}+\sum_{\lambda_{k} \geq 0, \forall k}^{n} y_{j k} \lambda_{k} \geq 0, \forall j
$$

em que Effo ou $h$ : eficiência da DMU o em análise

$\lambda_{k}$ : $\quad$ contribuição da DMU k na formação do alvo da DMU o

xik e yjk: inputs i e outputs $\mathrm{j}$ da DMU $\mathrm{k}, \mathrm{k}=1, \ldots, \mathrm{n}$

xio e jio: inputs i e outputs j da DMU o

O modelo BCC proposto por Banker et al. (1984) é também conhecido como VRS (Variable Returns to Scale), uma vez que considera situações de eficiência de produção com variação de escala, substituindo o axioma da proporcionalidade entre inputs e outputs pelo da convexidade. Dessa forma, deixa de obedecer a propriedade do raio ilimitado. Uma distorção do modelo VRS 
é considerar sempre eficientes as DMUs que tenham o menor valor para um dos inputs ou o maior valor para um dos outputs (Soares de Mello et al., 2004). A elas é dado o nome de eficientes por default.

\section{MÉTODO DE PESQUISA}

\subsection{Seleção de variáveis em DEA}

Segundo Senra et al. (2004), a metodologia DEA, historicamente, possui a fragilidade de sua baixa capacidade em ordenar DMUs, uma vez que, ao se calcular um modelo com muitas variáveis em relação ao número de unidades tomadoras de decisão, muitas são classificadas como eficientes.

Na literatura DEA, a grande maioria dos trabalhos publicados traz a abordagem da seleção de variáveis segundo a opinião de especialistas ou até mesmo da disponibilidade de dados (Senra et al., 2007). Segundo Lins e Angulo Meza (2000), quando se dispõe de poucas variáveis e muitas DMUs, a utilização de todas as variáveis não prejudica o modelo. Logo, não se justifica o cuidado em se valer de técnicas de seleção em variáveis.

Por outro lado, caso o número de DMUs seja pequeno, utilizar muitas variáveis pode diminuir o poder discriminatório do modelo e classificar muitas como eficientes. Uma solução para este caso é restringir as variáveis que serão analisadas, fazendo uso de um dos métodos de seleção de variáveis, objetivando aumentar a possibilidade de que apenas os inputs e os outputs mais adequados sejam utilizados na avaliação de eficiência.

Senra et al., 2007 realizam um estudo sobre métodos de seleção de variáveis em DEA no qual discorrem sobre as metodologias I-O Stepwise Exaustivo Completo, Multicritério para Seleção de Variáveis em Modelos DEA, Multicritério Combinatório Inicial para Seleção de Variáveis, Multicritério Combinatório por Cenários para Seleção de Variáveis.

\subsection{Método Multicritério para Seleção de Variáveis em Modelos DEA}

Neste estudo, optou-se pela utilização do Método Multicritério para Seleção de Variáveis em Modelos DEA proposto por Soares de Mello et al. (2002) por permitir a escolha inicial do par de variáveis pelo decisor, combinar uma relação causal entre inputs e outputs satisfatória, além de garantir a discriminação entre as DMUs. A aplicação deste método pode ser vista em Senra e Soares de Mello (2004) em análises do setor elétrico.

0 método de seleção constitui na adesão dos passos descritos a seguir:

I. Escolher um par input-output inicial: o decisor escolhe o melhor par input-output para iniciar a análise, baseando-se no seu conhecimento sobre o assunto e no tipo de medida que pretende fazer.

II. Calcular a eficiência média para cada variável acrescentada: os valores obtidos das eficiências médias serão normalizados com o uso de uma interpolação que atribua valor 1 à maior eficiência média e 0 à menor. Este valor normalizado é chamado SEF.

III. Contar o número de DMUs na fronteira para variável acrescentada: este número será normalizado com o uso de uma interpolação que atribua valor 1 ao menor número de DMUs na fronteira e 0 ao maior número. Observa-se que esta normalização garante que quanto maior for o número de DMUs na fronteira, menor será o poder discriminatório do modelo. Para tais valores é dado o nome de SDIS.

IV. Fazer uma soma ponderada de SEF e SDIS: com restrição de que a soma dos pesos seja 
igual a 1, esta soma é equivalente a uma soma ponderada, cabe ao decisor escolher o peso de cada variável de acordo com o fator que ele julgar mais relevante. Foi assim definida a variável $S=\alpha \mathrm{SEF}+(1-\alpha)$ SDIS.

V. Escolher a variável que tenha maior valor de $\mathrm{S}$ : a alternativa que tiver o maior valor de $\mathrm{S}$ é considerada que melhor concilia uma boa ordenação (alto valor de SDIS) e uma boa relação causal (alto valor de SEF), e será incluída no modelo.

VI. Verificar se o número de DMUs excede o quíntuplo do número de variáveis: caso afirmativo, deve-se encerrar a seleção de variáveis. Caso contrário, deve-se reiniciar o processo na etapa 2 incluindo mais uma variável. É importante ressaltar que a escolha de 1/5 é arbitrária e baseada em estudos de Gonzalez-Araya (2003).

\subsection{Fronteira Invertida}

O conceito de Fronteira Invertida foi introduzido por Yamada et al. (1994) e posteriormente ilustrado por Entani et al. (2002), os quais denominavam o método de IDEA ("Inverted DEA"). Seu uso para contornar problemas de baixa discriminação foi proposto inicialmente por Angulo Meza et al. (2003).

A Fronteira Invertida é uma avaliação pessimista das DMUs. Dessa forma é feita uma troca entre inputs com os outputs do modelo original. Esta fronteira é composta pelas DMUs com as piores práticas gerenciais podendo ser chamada de fronteira ineficiente (Leta et al., 2005).

Por consequência, o método impede a especialização das DMUs quando as mesmas atribuem pesos nulos às variáveis em que não apresentam resultado satisfatório para que não tenham suas eficiências prejudicadas. A Fronteira Invertida anula esse efeito, destacando também variáveis nas quais as DMUs não possuem bom desempenho. (Soares de Mello et al.,2005).

0 cálculo da eficiência invertida permite o cálculo da eficiência composta que considera tanto a avaliação otimista da fronteira padrão, quanto a pessimista da fronteira invertida. Segundo Leta et al. (2005), o índice composto à DMU mais eficiente é aquele que consegue ter um bom desempenho naquilo em que é melhor sem ter um desempenho ruim naquilo em que é pior.

0 índice de eficiência composta, como observado na equação (7), é definido pela média aritmética entre a eficiência em relação à fronteira convencional e o complemento da eficiência em relação à fronteira invertida.

$$
\text { Eficiência }{ }_{\text {composta }}=\frac{\text { Eficiência }_{\text {padrão }}+\left(1-\text { Eficiência }_{\text {invertida })}\right.}{2}
$$

Aplicações deste método podem ser encontrados em Maciel et al. (2014), na qual é realizada uma análise no processo de licitatório de contratação de embarcações, e também em Reinas et al. (2011), na qual são realizados cálculos para custo/benefício de aeronaves.

\section{OBJETO DE ESTUDO}

Os usuários interagem diretamente com os funcionários alocados nas estações. A alocação desses funcionários entre as estações deve permitir condições de trabalho satisfatórias pela ótica dos empregados, evitando a sobrecarga dos mesmos. Ao mesmo tempo, deve evitar o desperdício de recursos como a subutilização do capital humano quando há excesso de mão de obra para execução de tarefas necessárias na rotina das estações.

A percepção pelo passageiro da qualidade do serviço prestado se dá por quesitos como ingresso nas estações sem esperas significativas, a disponibilidade fácil de informações, a segurança e, limpeza do ambiente e a pontualidade de saída do trem de uma estação até a de destino. 
A via férrea do Rio de Janeiro tem extensão de $270 \mathrm{~km}$ que atendem a 102 estações de 5 ramais e 3 extensões. Servindo a 12 municípios da região metropolitana, são transportados uma média de 700 mil passageiros por dia útil em uma frota de 201 trens em operação.

O objetivo deste presente estudo é calcular as eficiências das estações ferroviárias do Ramal Deodoro em relação a utilização de sua mão de obra e passageiros atendidos, a fim de viabilizar uma análise de melhores práticas nas estações e melhor alocação de mão obra utilizada.

O Ramal Deodoro possui aproximadamente $22 \mathrm{~km}$ de extensão atendendo a 20 estações. A escolha deste ramal para análise se justifica pelo mesmo concentrar aproximadamente $48 \%$ do total de passageiros que utilizam o sistema. Sendo assim, qualquer melhoria de utilização ou redução de recursos apresentará resultados mais significativos do que se realizado em qualquer outro ramal.

\subsection{Análise de dados e aplicação do modelo}

Para a realização do estudo foi necessária a coleta inicial dos dados apresentados na Tabela 1. Foram excluídas da análise as estações Central do Brasil e Mangueira. 0 primeiro caso se deu para que não houvesse interferências na análise ao comparar uma estação que, por ser interseção entre todos os ramais, atende a um número muito maior de passageiros do que as outras estações menores. Já Mangueira não foi considerada na análise por impossibilidade de acesso aos dados operacionais.

As variáveis abaixo foram selecionadas pelo método de pesquisa adotado pois refletem como características operacionais das estações, como o número de linhas e viagens, interferem na quantidade de passageiros que frequentam o espaço tornando necessária a presença de mais funcionários. Já o número de pagantes na bilheteria, ou seja, pessoas que não utilizam cartões de transporte pré-carregados, influencia diretamente na quantidade de bilheteiros.

Tabela 1: Dados utilizados de total de passageiros e pagantes nas bilheteiras em dias úteis, quantidade de viagens, total de funcionários e número de linhas distribuídos por estações

\begin{tabular}{|c|c|c|c|c|c|}
\hline Estação & Passageiros em dias úteis & Pagantes na bilheteria & Viagens & Total Funcionários & Número de linhas \\
\hline Bento Ribeiro & 7093 & 2534 & 208 & 20 & 3 \\
\hline Cascadura & 8228 & 2780 & 208 & 20 & 4 \\
\hline Deodoro & 15000 & 4161 & 523 & 62 & 12 \\
\hline Engenho Novo & 8641 & 3054 & 208 & 16 & 2 \\
\hline Engenho de Dentro & 23273 & 7673 & 522 & 38 & 5 \\
\hline Madureira & 69988 & 20378 & 522 & 72 & 5 \\
\hline Maracanã & 27410 & 9520 & 783 & 38 & 6 \\
\hline Marechal Hermes & 12021 & 4154 & 208 & 18 & 4 \\
\hline Méier & 29642 & 10569 & 208 & 32 & 2 \\
\hline Oswaldo Cruz & 6547 & 2265 & 208 & 18 & 4 \\
\hline Praça da Bandeira & 9366 & 3035 & 208 & 18 & 2 \\
\hline Piedade & 6860 & 2560 & 208 & 14 & 2 \\
\hline Quintino & 5808 & 1911 & 208 & 14 & 2 \\
\hline Riachuelo & 9199 & 2778 & 208 & 14 & 2 \\
\hline São Francisco Xavier & 5554 & 1717 & 208 & 24 & 4 \\
\hline Sampaio & 3994 & 1581 & 208 & 18 & 2 \\
\hline São Cristóvão & 60223 & 18242 & 783 & 52 & 6 \\
\hline Silva Freire & 1348 & 473 & 77 & 4 & 2 \\
\hline
\end{tabular}

Os dados operacionais foram coletados e compilados a partir da empresa gestora da ferrovia. Os dados utilizados sobre passageiros e pagantes nas bilheteiras correspondem à média de dias úteis entre maio de 2016 até maio de 2017 multiplicadas por um fator de correção para preser- 
var o sigilo dos dados. A alteração não apresenta danos ao modelo uma vez que a Análise Envoltória de Dados é invariante à escala.

Ser invariante à escala significa que os resultados finais da análise não se alterariam se, em um exemplo hipotético, uma variável fosse apresentada em metros ou em centímetros. No caso deste estudo, ser invariante à escala significa que obteremos os mesmos resultados calculando o modelo com dados reais ou multiplicando todos os valores da variável pelo valor hipotético X. O número de viagens e o número de linhas correspondem ao cenário realista em maio de 2017. 0 total de funcionários foi estimado de acordo com o observado nas estações e corresponde ao número de empregados alocados nos cargos de bilheteiros, agentes de segurança, auxiliadores de público e profissionais de limpeza.

O número de viagens corresponde a quantas vezes o trem para na estação de modo que haja embarque e desembarque de passageiros, não importando o sentido. 0 número de linhas corresponde ao número de vias em que há a possibilidade do Material Rodante passar pela estação. Usualmente, as estações possuem pelo menos duas linhas de mesma direção, porém com sentidos de movimentação opostos. As estações que possuem mais de duas linhas em geral estão em locais nos quais a via permanente pode atender a mais de um ramal. Vale ressaltar que a conexão entre os ramais nestes pontos depende da operação da empresa que obtém a concessão da linha férrea.

Com o intuito de garantir a ótica do modelo voltada para a mão de obra utilizada, optou-se pela utilização da mesma como único input a ser reduzido utilizando a orientação voltada a input. Por opção do decisor em manter a relação mão de obra e passageiros, a utilização do par inicial de variáveis se deu da seguinte forma:

Input inicial: Total de Funcionários

Output inicial: Passageiros em dias úteis

A seleção de outras variáveis foi realizada utilizando o método de Multicritério para Seleção de Variáveis em Modelos DEA. Além disso, para todos os cálculos do modelo foi utilizado o modelo CCR orientado a input, com o objetivo de evitar que sejam colocadas na fronteira de eficiência as DMUs eficientes por default apresentadas do modelo BCC, que seriam aquelas que apresentam o menor número de funcionários ou que atendem ao maior número de passageiros e linhas.

Neste trabalho será utilizado o modelo CCR com orientação a inputs e cálculo da Fronteira Invertida para a análise de eficiência na alocação de mão de obra nas estações ferroviárias.

Para a seleção da terceira variável foram calculados 3 modelos utilizando o par inicial de variáveis e acrescentados das variáveis Pagantes, Viagens e Linhas individualmente na posição de output. Para os cálculos de eficiência, foi utilizado o Software de Sistema Integrado de Apoio à Decisão (SIAD) desenvolvido por Angulo Meza et al. (2005a, 2005b).

Na sequência, seguindo o modelo de seleção de variáveis, foram calculadas as eficiências médias de cada modelo, assim como os valores de SEF e SDIS que viabilizaram o cálculo de S utilizando $\alpha=0,5$ igualando, portanto, as prioridades dadas ao cálculo de eficiência e discriminação, conforme apresentado na Tabela 2.

A variável que apresentou o maior valor de $S$ foi a quantidade de linhas e a mesma foi incluída no modelo. Como são 18 DMUs utilizadas, optou-se por encerrar o processo de seleção para que a regra 1/5, apresentada na seção de seleção de variáveis, não fosse ultrapassada. 
Tabela 2: Cálculo de eficiência para seleção de variáveis

\begin{tabular}{llll}
\hline DMU & Pagantes & Viagens & Linhas \\
\hline Bento Ribeiro & 0,361167 & 1,000000 & 0,480562 \\
Cascadura & 0,396228 & 1,000000 & 0,596938 \\
Deodoro & 0,208898 & 0,233847 & 0,466473 \\
Engenho Novo & 0,544102 & 0,483197 & 0,574582 \\
Engenho de Dentro & 0,575514 & 0,543652 & 0,636057 \\
Madureira & 0,839315 & 0,844382 & 0,839315 \\
Maracanã & 0,714141 & 0,634711 & 0,753593 \\
Marechal Hermes & 0,657847 & 0,590034 & 0,813338 \\
Méier & 0,941399 & 0,806131 & 0,799818 \\
Oswaldo Cruz & 0,358538 & 0,335640 & 0,596733 \\
Praça da Bandeira & 0,480479 & 0,466647 & 0,539377 \\
Piedade & 0,521246 & 0,441282 & 0,566038 \\
Quintino & 0,389306 & 0,378446 & 0,512536 \\
Riachuelo & 0,567282 & 0,580929 & 0,684941 \\
São Francisco Xavier & 0,203815 & 0,225051 & 0,418120 \\
Sampaio & 0,250533 & 0,217084 & 0,326886 \\
São Cristóvão & 1,000000 & 1,000000 & 1,000000 \\
Silva Freire & 0,337792 & 0,313341 & 1,000000 \\
\hline Eficiência Média & 0,519311 & 0,560799 & 0,644739 \\
DMUS na fronteira & 1,000000 & 3,000000 & 2,000000 \\
SEF & 0,000000 & 0,330768 & 1,000000 \\
SDIS & 1,000000 & 0,000000 & 0,500000 \\
S & 0,500000 & 0,165384 & $\mathbf{0 , 7 5 0 0 0 0}$ \\
\hline
\end{tabular}

Por mais que o número de linhas atendidas pelas estações possa ser considerado um input e fator relevante para a decisão de um passageiro em acessar ou não o sistema naquele ponto da malha ferroviária, a utilização da variável selecionada como output se dá pelo sentido dado ao modelo proposto que visa a melhoria na alocação do quadro de funcionários. Além disso, não é interessante para os passageiros que o número de linhas venha a ser reduzido.

Dessa forma, para o cálculo das eficiências para cada estação foi utilizado o modelo CCR orientado a input utilizando as variáveis listadas abaixo:

Input: Total de Funcionários

Output: Passageiros em dias úteis e número de linhas

Analisando as eficiências padrão previamente calculadas para as estações durante a fase de seleção de variáveis e apresentadas na Tabela 3, pode-se perceber que as únicas DMUs que se encontram na fronteira são São Cristóvão e Silva Freire.

Tabela 3: Cálculo de eficiência padrão

\begin{tabular}{ll}
\hline DMU & Eficiência Padrão \\
\hline Bento Ribeiro & 0,480562 \\
Cascadura & 0,596938 \\
Deodoro & 0,466473 \\
Engenho Novo & 0,574582 \\
Engenho de Dentro & 0,636057 \\
Madureira & 0,839315 \\
Maracanã & 0,753593 \\
Marechal Hermes & 0,813338 \\
Méier & 0,799818 \\
Oswaldo Cruz & 0,596733 \\
Praça da Bandeira & 0,539377 \\
Piedade & 0,566038 \\
Quintino & 0,512536 \\
Riachuelo & 0,684941 \\
São Francisco Xavier & 0,418120 \\
Sampaio & 0,326886 \\
São Cristóvão & 1 \\
Silva Freire & 1 \\
\hline
\end{tabular}


Os resultados encontrados através do cálculo do Problema de Programação Linear (PPL) são confirmados pelo Gráfico $1 \mathrm{em}$ que seus eixos foram elaborados por meio da divisão dos outputs pelo input. Assim:

- Eixo horizontal: divisão do Número de Linhas pelo Total de Funcionários

- Eixo vertical: divisão dos Passageiros em dias úteis / Total de Funcionários

A fronteira de eficiência, representada pela linha contínua no Gráfico 1, é dada pela estação mais distante do eixo horizontal (São Cristóvão) e por outra mais distante do eixo vertical (Silva Freire). Caso houvesse outras DMUs eficientes, elas fariam parte da fronteira de eficiência e se apresentariam graficamente na reta que liga São Cristóvão a Silva Freire ou acima dela, modificando o formato da fronteira.

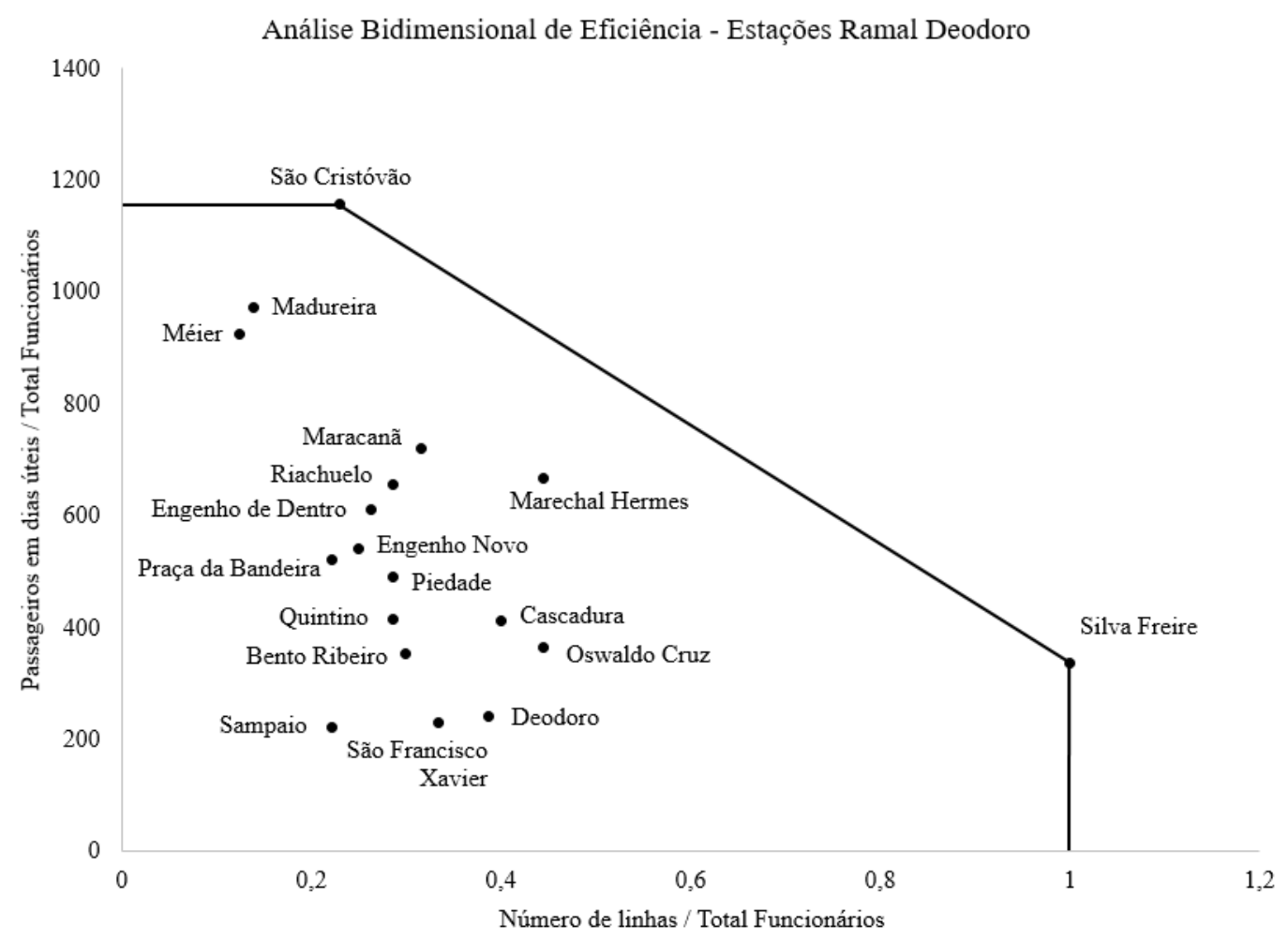

Gráfico 1. Análise bidimensional de eficiência

Tabela 4: Cálculo de benchmarks

\begin{tabular}{lll}
\hline DMU & São Cristóvão & Silva Freire \\
\hline Bento Ribeiro & 0,090281 & 0 \\
Cascadura & 0,098469 & 0 \\
Deodoro & 0,123033 & 0 \\
Engenho Novo & 0,129833 & 0 \\
Engenho de Dentro & 0,354254 & 0 \\
Madureira & 1,162128 & 0 \\
Maracanã & 0,415913 & 0 \\
Marechal Hermes & 0,166002 & 0 \\
Méier & 0,492196 & 0 \\
Oswaldo Cruz & 0,068530 & 0 \\
Praça da Bandeira & 0,142720 & 0 \\
Piedade & 0,098113 & 0 \\
Quintino & 0,079388 & 0 \\
Riachuelo & 0,139729 & 0 \\
São Francisco Xavier & 0,050872 & 0 \\
Sampaio & 0,047099 & 0 \\
São Cristóvão & 1,000000 & 0 \\
Silva Freire & 0,000000 & 0 \\
\hline
\end{tabular}


O fato de São Cristóvão e Silva Freire estarem na fronteira de eficiência significa que ambos podem ser considerados benchmarks para as outras DMUs. Todavia, ao analisar os valores encontrados pelo cálculo de benchmark, pode-se verificar que todas as estações não consideram Silva Freire como um alvo, inclusive ela mesma, conforme Tabela 4.

Em busca de uma análise mais profunda e da possibilidade de ordenar as estações por eficiência, foi realizado o cálculo de Fronteira Invertida que, como dito anteriormente, inverte outputs e inputs permitindo a análise das DMUs ineficientes. Como pode-se notar pelos resultados da Tabela 5, a estação Méier e Sampaio se encontram na fronteira de ineficiência, e as estações Madureira, Deodoro e São Francisco Xavier muito próximas a ela, com ineficiências acima de $90 \%$.

Tabela 4: Cálculo de benchmarks

\begin{tabular}{lllll}
\hline DMU & Eficiência Padrão & Eficiência Invertida & Eficiência Composta & Eficiência Composta Normalizada \\
\hline São Cristóvão & 1 & 0,647306 & 0,676347 & 1 \\
Marechal Hermes & 0,813338 & 0,471180 & 0,671079 & 0,992211 \\
Silva Freire & 1 & 0,658424 & 0,670788 & 0,991781 \\
Maracanã & 0,753593 & 0,608766 & 0,572414 & 0,846331 \\
Cascadura & 0,596938 & 0,553541 & 0,521699 & 0,771347 \\
Riachuelo & 0,684941 & 0,671765 & 0,506588 & 0,749006 \\
Oswaldo Cruz & 0,596733 & 0,610144 & 0,493295 & 0,729351 \\
Madureira & 0,839315 & 0,926013 & 0,456651 & 0,675173 \\
Engenho de Dentro & 0,636057 & 0,727235 & 0,454411 & 0,671861 \\
Piedade & 0,566038 & 0,715585 & 0,425226 & 0,628710 \\
Méier & 0,799818 & 1 & 0,399909 & 0,591278 \\
Engenho Novo & 0,574582 & 0,779077 & 0,397752 & 0,588089 \\
Quintino & 0,512536 & 0,737223 & 0,387656 & 0,573162 \\
Bento Ribeiro & 0,480562 & 0,724583 & 0,377990 & 0,558869 \\
Praça da Bandeira & 0,539377 & 0,859914 & 0,339731 & 0,502303 \\
Deodoro & 0,466473 & 0,917141 & 0,274666 & 0,406102 \\
São Francisco Xavier & 0,418120 & 0,958828 & 0,229646 & 0,339539 \\
Sampaio & 0,326886 & 1 & 0,163443 & 0,241655 \\
\hline
\end{tabular}

Analisando a eficiência composta, ou seja, a combinação entre valores de eficiência e ineficiência, apenas as DMUs Cascadura, Maracanã, Marechal Hermes, Riachuelo, São Cristóvão e Silva Freire apresentaram valores acima de 0,5. Neste caso, as outras DMUs se aproximam mais da fronteira de ineficiência do que de eficiência.

Calculando os valores da composta normalizada, é possível ordenar as DMUs por eficiência. Tal ordenação pode ser vista na tabela 5 .

\section{CONCLUSÕES}

Após os resultados encontrados, pode-se constatar que, analisando os valores da eficiência composta, as estações de Cascadura, Maracanã, Marechal Hermes, Riachuelo, São Cristóvão e Silva Freire apresentam-se mais próximas da fronteira de eficiência enquanto as demais se aproximam mais da fronteira de ineficiência. No entanto, somente a estação de São Cristóvão apresenta-se eficiente a ponto de ser considerada benchmark para todas as demais estações.

Dessa forma, as práticas e critérios adotados na utilização de mão de obra na estação de São Cristóvão devem ser, quando operacionalmente possível, replicados e/ou adaptados às demais estações, a fim de que possa ser melhorada a eficiência global do atendimento aos passageiros usuários da Ramal de Deodoro, por meio de uma melhor otimização de seus recursos.

A ordenação das estações pode ser considerada uma ferramenta de auxílio para o desenvolvimento de um plano de ação para análise e melhorias da utilização de mão de obra das estações iniciando pelas menos eficientes. 
Independentemente dos resultados aqui apresentados e da ampla revisão de literatura, este estudo não tem a pretensão de exaurir todo o tema, disponibilizando informações que possam ser adotadas por outros estudos futuros, os quais possam incluir variáveis não consideradas no presente trabalho, assim como dados mais recentes à época das novas iniciativas.

Além disso, é indicado para futuras análises avaliar a adequação do modelo CCR ao objeto de estudo, a utilização do modelo BCC, a inclusão de outras DMUs e a explicação do fato da Estação Silva Freire não possuir benchmarks.

\section{REFERÊNCIAS}

Angulo Meza, L.; L. Biondi Neto, J. C. C. B. Soares de Mello e E. G. Gomes (2005a) ISYDS - Integrated System fo Decision Support (SIAD - Sistema Integrado de Apoio à Decisão): a software package for data envelopment analysis model. Pesquisa Operacional, v. 25, n. 3, P. 493-503.

Angulo Meza, L.; L. Biondi Neto, J. C. C. B. Soares de Mello, E. G. Gomes, P. H. G. Coelho (2005b) Free software for decision analysis: a software package for data envelopment models. 7th International Conference on Enterprise Information Systems - ICEIS, v. 2, P. 207-212.

Caldas, M. A. F.; P. D. Gabriele, R. L. Carvalhal e T. G. Ramos (2012) A Eficiência do Transporte Ferroviário de Cargas: Uma Análise do Brasil e dos Estados Unidos. Congresso Latino Ibero-Americano de Investigación Operativa e Simpósio Brasileiro de Pesquisa Operacional XVI CLAIO - XLIV SBPO, p. 1775-1786.

Charnes, A.; W. Cooper e E. Rhodes (1978) Measurind the efficiency of decision-making units. European Journal of Operational Research, v.2, n. 6, P. 429-444.

Himola, O. (2010) Building efficiency from bottom-up? Analysing global railway freight. International Jounal of Logistics Economics and Globalisation, v. 2, n. 2, P. 139-150.

Kutlar. A., K. Ali e M. Sarikaya (2013) Determination of the efficiency of the world railway companies by method of DEA and comparison of their efficiency by Tobit analysis. Quality \& Quantity, v. 47, n. 6, P. 3575-3602.

Lan, L. W. e E. T. Lin (2003) Technical efficiency and service effectiveness for railways industry: DEA approaches. Journal of the Eastern Asia Society for Transportation Studies, v. 5, p. 2932-2947.

Leta, F. R.; J. C. C. B. Soares de Mello, E. G. Gomes e Angulo Meza, L. (2005) Métodos de Melhora de Ordenação em DEA Aplicados à Avaliação Estática de Tornos Mecânicos. Investigação Operacional, v. 25, n. 2, P. 229-242.

Maciel, G. S.; G. B. A. Lima,L. Angulo Meza e S. F. Gomes Júnior (2014) Avaliação de processos licitatórios de embarcações de apoio marítmo offshore com utilização de análise envoltória de dados. Journal of Transport Literature, v. 8, n. 4, p. 329349.

Oum, T. H. e C. Yu (1994) Economic Efficiency of Railways and Implications for Public Policy - A comparative Study of the OECD Countries Railways. Journal of Transport Economics and Policy, v. 28, n. 2, p. 121-138.

Pereira, L. A. M. e. J. C. C. B. Soares de Mello (2014). Eficiência técnica das concessionárias ferroviárias nacionais usando Análise Envoltória de Dados. Relatórios de Pesquisa em Engenharia de Produção, v. 14, P. 246-256.

Pereira, M. A. R. F. S. e. L. R. J., 2015. Análise da eficiência ferroviária no Brasil nos anos entre 2009 a 2013. Transportes, v. 23 , n. 3, P. 56-63.

Reinas, R. I.; E. B. Mariano e D. A. N. Rebelatto (2011) Custo/benefício de aeronaves: uma abordagem pela Análise Envoltória de Dados. Produção, P. 684-695.

Reis, J. C.; K. T. Sacramento, J. C. C. B. Soares de Mello e L. Angulo Meza (2017) Avaliação de eficiência das ferrovias brasileiras: Uma aplicação do método multicritério para seleção de variáveis em DEA e Representação Gráfica Bidimensional. Revista Espacios, v. 38, n. 14, P. 16-26

Rubem, A. P. S.; J. C. C. B. Soares de Mello, L. Ângulo Meza e F. F. Gomes Júnior (2013) Análise de Eficiência de Companhias Aéreas com um modelo DEA e Clusters Dinâmicos. Journal of Transport Literature, vol. 10, n. 1, P. 20-24.

Sameni, M. K.; J. Preston e M. K. Sameni (2016) Evaluating efficiency of passengers railway stations: A DEA approach. Research in Transportation Business \& Management, v. 20, P. 33-38

Senra, L. F. A. C.; L. C. Nanci e J. C. C. B. Soares de Mello (2004) Métodos totais de seleção de variáveis em DEA. VII Simpósio de Pesquisa Operacional e Logística da Marinha, Niterói. Anais do VII SPOLM

Senra, L. F. A. C.; L. C. Nanci, L. C., J. C. C. B. Soares de Mello e L. Angulo Meza (2007) Estudo sobre métodos de Seleção de Variáveis em DEA. Pesquisa Operacional, v. 27, n. 2, P. 191-207.

Senra, L. F. A. C. e J. C. C. B. Soares de Mello (2004). Uso de técnicas de seleção de variáveis em DEA para analisar o setor elétrico. Relatório de Pesquisa em Engenharia de Produção, v. 4, p. 4.

Yu, M. M. (2008). Assessing the technical efficiency, service effectiveness, and technical effectiveness os the world`s railways through NDEA analysis. Transportation Research Part A: Policy and Practice, v. 42, n. 10, P. 1283-1294. 